\title{
Editorial. Meta-investigación en comunicación: antecedentes, efectos y retos de una investigación y gobernanza estandarizada
}

\author{
Editorial. Meta-research in communication: \\ antecedents, effects, and challenges of a standardized \\ research and governance
}

\author{
Manuel Goyanes
}

Cómo citar este artículo:

Goyanes, Manuel (2020). "Meta-investigación en comunicación: antecedentes, efectos y retos de una investigación y gobernanza estandarizada". Profesional de la información, v. 29, n. 4, e290406.

https://doi.org/10.3145/epi.2020.jul.06

Artículo invitado recibido el 13-06-2020

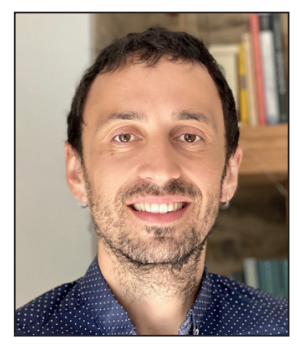

\author{
Manuel Goyanes \\ https://orcid.org/0000-0001-6537-9777 \\ Universidad Carlos III de Madrid \\ Departamento de Periodismo y \\ Comunicación Audiovisual \\ Madrid, 133 \\ 28903 Getafe (Madrid), España \\ mgoyanes@hum.uc3m.es
}

\section{Resumen}

Históricamente, la meta-investigación en comunicación tanto en España como internacional se ha focalizado en la presión de producción (autores), examinando detalladamente los patrones de creación científica. Muy limitados han sido sin embargo los esfuerzos orientados a problematizar el modelo de creación de oferta y, más concretamente, el sistema de gobernanza que sustenta, legitima y estimula el paradigma de producción tal cual se presenta: los comités científicos. En este ensayo se razona sobre la relevancia de los consejos editoriales en comunicación y se revisan sus efectos. Específicamente, este artículo editorial inicia y delinea un programa de investigación con el que comprender mejor las desigualdades y oposiciones de la gobernanza académica y sus efectos directos e indirectos en la producción científica, describiendo las teorías más destacadas para su examen. Finalmente, se plantean los retos del futuro de la meta-investigación en comunicación en España e internacional.

\section{Palabras clave}

Comunicación; Comités científicos; Consejos editoriales; Meta-investigación; Gobernanza; Pluralismo; Producción científica; Revistas científicas; Comunicación académica.

\begin{abstract}
Meta-research in communication has extensively documented the main patterns of scientific production, yet scant attention has been paid to the governance structure that underpins, legitimates, and stimulates the current scientific paradigm: journals' editorial boards. This essay examines the relevance of editorial boards in communication and illustrates their potential productive effects. Specifically, this editorial article initiates a research program aiming at better understanding the inequalities in editorial boards and their direct and indirect effects at the level of research production, outlining the main theories for their examination. Finally, based on this thematic summary, the essay offers suggestions on how to move forward and identify some of the main challenges and gaps in this promising area of inquiry.
\end{abstract}

\section{Keywords}

Communication; Editorial boards; Scientific committees; Meta-research; Governance; Pluralism; Scientific production; Scientific journals; Scholarly communication. 


\section{Introducción}

La investigación en el campo de la comunicación ha sido tradicionalmente un espacio diverso y multidisciplinar, con múltiples escuelas, tradiciones y aproximaciones a la miríada de objetos que componen cada sub-disciplina (Craig, 1999; Donsbach, 2006; Fuchs; Qiu, 2018). Esta diversidad en las aproximaciones y procedimientos de investigación ha resultado en un campo de investigación relativamente disperso, espeso y sin una definición acotada y compartida sobre los principales objetos que regulan este terreno intelectual (Miconi; Serra, 2019). Sin embargo, durante las últimas décadas y especialmente en España, la investigación en el campo de la comunicación ha experimentado una profunda transformación (Martínez-Nicolás; Saperas-Lapiedra, 2016), fruto de la asimilación del establishment académico de los principios y orientaciones que gobiernan las carreras académicas y la consolidación de rankings y listas de clasificación dominadas por la Communication Research (Goyanes, 2017).

A pesar de la existencia de grandes espacios intelectuales que mantienen sus propias normas y valores de producción y evaluación, son cada vez más las presiones para establecer un régimen científico acumulativo y a semejanza de las ciencias duras, lo que desencadena grandes desafíos que afectan a la definición del propio ethos de la investigación en comunicación y al encaje de la diversidad, las escuelas académicas y las tradiciones teóricas en los circuitos de difusión de la "excelencia" (Goyanes, 2017; Martínez-Nicolás; Saperas; Humanes, 2019). En este contexto, la problematización del estado de la investigación en el campo de la comunicación y la propuesta de reflexiones que razonen sobre las estructuras que regulan y gobiernan el modo de producción científica en España y en el mundo, resulta esencial. Este número especial trata de reflexionar y problematizar los mecanismos que regulan la investigación en comunicación con el objetivo de plantear nuevos escenarios para el desarrollo y encaje de la diversidad, la originalidad y la innovación. En este sentido, uno de los espacios clave para entender la estructura de las desigualdades y los mecanismos de reproducción científica son los órganos de gobernanza, es decir, los consejos científicos (Goyanes; Demeter, 2020).

\section{Detección del problema y estructura del ensayo}

La bibliografía existente sobre cienciometría o meta-investigación en comunicación en España (Fernández-Quijada; Masip; Bergillos, 2013; Rodrigo-Alsina; García-Jiménez, 2010; Caffarel-Serra; Ortega-Mohedano; Gaitán-Moya, 2017) e internacional (Bunz, 2005; Günther; Domahidi, 2017; Walter; Cody; Ball-Rokeach, 2018; Keating et al., 2019), se ha centrado tradicionalmente en el lado de la fuerza de producción, estudiando con gran detalle los investigadores y sus redes de coautoría, los patrones de citación y las tendencias de publicación más relevantes. Sin embargo, estos estudios han obviado sistemáticamente el lado de la oferta y los mecanismos estructurantes que vehiculan los modelos y esquemas de cientificidad: los consejos científicos. ¿Cómo afecta la diversidad geográfica de los comités científicos en lo que las revistas publican? ¿De qué manera los miembros de los comités editoriales se estructuran en torno a redes-élite? $Y$ sobre todo: ¿cómo se estructuran los colegios invisibles y qué impacto tienen en la producción de conocimiento? Este artículo editorial traza una línea de investigación sobre la influencia de los consejos editoriales en la estructuración del campo de la comunicación, enfatizando su relevancia como mecanismo indirecto para perpetuar la línea dominante de producción científica (Burgess; Shaw, 2010; Metz; Harzing; Zyphur, 2016).

Este editorial se estructura del siguiente modo: en primer lugar se definen las funciones y modelos de reclutamiento de los comités científicos para luego problematizar los efectos de su distorsionada composición geográfica y de género. A continuación, a partir de la teoría de las membresías cruzadas se da cuenta de la estructura elitista que determina las normas y valores que gobiernan el sistema científico en comunicación. Una vez descrita esta red de relaciones, se describen las teorías más destacadas con las que examinar los desequilibrios y desigualdades en comunicación para, finalmente, razonar sobre los retos y desafíos más apremiantes de la meta-investigación en España e internacional.

\section{Gobernanza en la ciencia: Ios comités editoriales como "guardianes del conocimiento"}

En las últimas décadas las revistas de investigación se han convertido en uno de los espacios más influyentes para la difusión de avances científicos en la mayoría de disciplinas del mundo (Pendlebury; Adams, 2012). Su creciente papel ha desencadenado una progresiva competencia entre académicos (Grimes; Bauch; loannidis, 2018), cada vez más preocupados por su historial de publicaciones y el grado en que sus estudios son considerados "excelentes" (Butler; Spoelstra, 2014). La publicación de investigaciones de calidad es la vocación esperada de la mayoría de investigadores, independientemente de su experiencia y geografía (Goyanes, 2017). Sin embargo, esta suposición es muy a menudo guillotinada por el proceso de revisión por pares, la base del progreso científico. Esta responsabilidad, entre otras muchas, es generalmente desempeñada por académicos pertenecientes a los consejos editoriales (Espin et al., 2017).

La limitada, pero cada vez más relevante bibliografía sobre los consejos editoriales ha abordado tres materias interrelacionadas: 1) las funciones, lógicas y objetivos de este cuerpo de gobernanza, 2) el proceso de reclutamiento y selección de sus miembros así como sus características individuales y, 3) los potenciales efectos de la falta de diversidad geográfica y de género (Burgess; Shaw, 2010; Espin et al., 2017; Metz; Harzing; Zyphur, 2016; Dhanani; Jones, 2017). En este artículo editorial se describen los hallazgos fundamentales que dan cuenta de las principales desigualdades y desequilibrios 
de los consejos editoriales, enfatizando los desafíos generados por la homogeneización de los perfiles geográficos. Por lo tanto, el enfoque se sitúa en la última corriente. Sin embargo, para obtener una mejor perspectiva sobre el papel de los consejos editoriales, se discuten inicialmente sus funciones (Willett, 2013) para luego problematizar los desequilibrios y desigualdades que su homogeneización desencadena (Goyanes, 2017; Demeter, 2018).

\section{Funciones y efectos de los consejos editoriales}

Los consejos editoriales son el cuerpo de gobernanza de las revistas científicas (Espin et al., 2017). La función de los consejos editoriales se manifiesta mediante la metáfora de "los guardianes del conocimiento" (Metz; Harzing; Zyphur, 2016), una idea que muestra su influencia en la estructuración de la producción científica y por lo tanto lo que determina el desarrollo de la teoría, la investigación y la práctica (Metz; Harzing, 2009). Como organismo institucional en el gobierno de las comunidades académicas (Dhanani; Jones, 2017), los comités editoriales desempeñan una función decisiva al delinear quién y qué merecen ser publicados (Goyanes; De-Marcos, 2020). Asimismo, comparten una influencia determinante en la progresión académica, señalando los problemas, prioridades y enfoques que se deben problematizar o discutir en detalle (García-Carpintero; Granadino; Plaza, 2010). Al hacerlo, brindan estatus y visibilidad a ciertos académicos, confiriendo autoridad y recomendando sus investigaciones (Willett, 2013). Además, proporcionan las estrategias, técnicas y tácticas necesarias para mejorar el desarrollo de las revistas (Burgess; Shaw, 2010), ocupando una posición destacada en el debate académico.

En resumen, los miembros de los consejos editoriales ayudan a los editores a mejorar la calidad de las revistas mediante tres mecanismos (Willett, 2013): 1) anunciando, promocionando y ofreciendo notoriedad a la revista y sus publicaciones, 2) sirviendo como revisores durante el proceso de revisión por pares ciego y 3 ) brindando asesoramiento técnico-táctico para el desarrollo de las revistas. Los consejos editoriales de las principales revistas son, en consecuencia, un elemento determinante en la configuración de los estándares de calidad de las revistas y, por tanto, en la progresión y legitimidad de los propios campos científicos (Parker, 2007).

Los miembros de los consejos editoriales son nombrados por los editores o editores jefe, generalmente mediante invitación (Dhanani; Jones, 2017). En la bibliografía relacionada se asume que para ser invitado a un consejo editorial los candidatos deben tener un amplio historial de publicaciones y ser considerados por sus pares como expertos de su disciplina (Petersen; Hattke; Vogel, 2017). En resumen, la investigación previa asume que la selección de consejeros científicos se basa en la trayectoria y excelencia de los candidatos, lo que generalmente implica la publicación continuada en las revistas más influyentes (Burgess; Shaw, 2010). Sin embargo, estudios recientes cuestionan la idea racional de la conexión causal entre excelencia y membresía. De acuerdo con Bedeian, Van Fleet y Hyman (2009), en el campo de la gestión no existe una correlación significativa entre la producción científica y la membresía a comités científicos, lo que sugiere que el argumento meritocrático no está respaldado por los datos y, en consecuencia, el "patronazgo" académico podría explicar mejor esta asociación (Burgess; Shaw, 2010).

La idea detrás del nombramiento de destacados académicos como consejeros editoriales se deriva de las posiciones privilegiadas que dichos perfiles ocupan como líderes de opinión (Crane, 1967). Por lo tanto, ser invitado a formar parte del comité científico de una prestigiosa revista es una forma fundamental de reconocimiento (Mauleón et al., 2013), favorablemente considerada en los procesos de evaluación y promoción académica (Metz; Harzing; Zyphur, 2016). Los nombres y cargos de los investigadores en la jerarquía del consejo editorial se muestran generalmente en las versiones impresas de las revistas y en sus webs oficiales (Burgess; Shaw, 2010). Por lo tanto, los consejeros científicos son generalmente visibles, aunque su proceso de reclutamiento no es suficientemente transparente (Ozbilgin, 2004). Estas sospechas sobre la composición de los consejos editoriales sientan la base de los recientes cuestionamientos sobre sus efectos productivos. En la siguiente sección se abordan estos desequilibrios en términos de diversidad de género y geográfica.

\section{Perspectivas sobre las desigualdades geográficas y de género en los consejos editoriales}

A pesar del potencial impacto de los consejos editoriales en la producción científica, la investigación formal sobre este cuerpo de gobernanza es todavía muy limitada (Burgess; Shaw, 2010; Mauleón et al., 2013; Dhanani; Jones, 2017). Las investigaciones existentes acerca de la composición de los comités científicos se han centrado fundamentalmente en dos cuestiones: su internacionalización (Burgess; Shaw, 2010; Willett, 2013; Goyanes, 2017) y los desequilibrios de género (Mauleón et al., 2013; Metz; Harzing; Zyphur, 2016). La suposición para promover estas investigaciones se basa en que la diversidad científica es un ingrediente clave para fomentar el pluralismo, permitiendo a las revistas diseñar espacios "con igualdad de oportunidades" (Dhanani; Jones, 2017, p. 3). Por lo tanto, se asume que al promover la diversidad en los consejos editoriales se estimulan los enfoques plurales sobre el progreso científico y se desarrollan estrategias inclusivas con las que fomentar el acceso a académicos no-occidentales (Goyanes, 2017). 
Algunas disciplinas han examinado la naturaleza de la gobernanza de sus revistas más que otras, pero en términos generales el dominio de los consejos editoriales por académicos hombres estadounidenses está bastante bien documentado en la mayoría de disciplinas, tales como la contabilidad (Dhanani; Jones, 2017), gestión (Burgess; Shaw, 2010), sistemas de información (Cabanac, 2012), ciencias políticas (Stegmaier; Palmer; Van-Assendelft, 2011), medicina (Morton; Sonnad, 2007), y comunicación (Demeter, 2018). El porcentaje de mujeres en los consejos editoriales varía en función de la revista y la disciplina, pero a lo largo de los años se documenta un aumento significativo en áreas como la epidemiología (Dickersin et al., 1998), la medicina (Sidhu et al., 2009) o la administración de empresas (Metz; Harzing; Zyphur, 2016). Sin embargo, a pesar de este notable incremento, el porcentaje de mujeres que forman parte de los consejos editoriales es todavía menor que el de mujeres autoras (Dickersin et al., 1998), lo que sugiere un techo de cristal en el que las minorías científicas (en este caso, mujeres, pero también autores del Sur-Global), se detienen en los peldaños inferiores de la escalera académica (Mauleón et al., 2013).

De igual modo, en la bibliografía relacionada, la diversidad geográfica de los consejos editoriales es un tema relevante. La suposición básica de esta línea de investigación parte de que la falta de diversidad geográfica en los consejos científicos amenaza la idea de las ciencias de la comunicación como una empresa global (Livingstone, 2007), lo que genera el establecimiento de conceptualizaciones teóricas occidentales como unidad de medida. Existe un amplio consenso en la bibliografía en que las revistas con una diversidad geográfica limitada restringen lo que se publica $y$, por lo tanto, legitiman los enfoques occidentales sobre cómo implementar y desarrollar investigación empírica (Braun; Diospatonyi, 2005).

\section{El porcentaje de mujeres que forman} parte de los consejos editoriales es todavía menor que el de mujeres autoras

Estudios previos en diferentes campos científicos han demostrado que los consejos editoriales están regidos por un puñado de geografías occidentales (Metz; Harzing; Zyphur, 2016; Demeter, 2018), siendo Estados Unidos el país más demandado (Burgess; Shaw, 2010; Willett, 2013; Goyanes, 2017). De acuerdo a estos estudios, la mayoría de revistas líderes están gobernadas casi exclusivamente por académicos del mundo desarrollado, mientras que las geografías del Sur-Global están fuertemente oscurecidas (Mauleón et al., 2013). Varios estudios evidencian este desequilibrio. Por ejemplo, en el campo de la gestión, los académicos y las principales universidades estadounidenses son los perfiles preponderantes (Burgess; Shaw, 2010). Al analizar la composición editorial de 16 de las revistas líderes de biblioteconomía y ciencias de la información, Willett (2013) demuestra que los hombres de Estados Unidos son el perfil prototípico. Demeter (2018), tras explorar la diversidad geográfica de las principales revistas en ciencias de la comunicación, denuncia que la mayoría de las principales revistas están regidas por académicos del mundo desarrollado. Haciéndose eco de estos hallazgos, Goyanes (2019), mostró que dos tercios de los miembros de los consejos editoriales de 39 revistas de comunicación en 2015 son de Estados Unidos, un porcentaje alarmante que contrasta con la gran diversidad internacional de la producción científica en esta disciplina. Y lo que es más importante, Goyanes y Demeter (2020), demuestran que estos patrones en la configuración de los comités editoriales tienen un efecto significativo en las metodologías empleadas y en el lugar de procedencia de los datos.

Por otra parte, los estudios bibliométricos han proporcionado evidencias cruciales para ilustrar el estado del campo y, por lo tanto, para calibrar indirectamente la potencial distorsión de la representación de los consejos editoriales (asumiendo que la publicación regular en revistas de primer nivel aumenta las probabilidades de ser un miembro elegible de sus órganos de gobernanza). A este respecto, una miríada de estudios ha analizado las principales tendencias y patrones de publicación en ciencias de la comunicación en la última década (Lauf, 2005; Chung et al., 2012; Knobloch-Westerwick; Glynn; Huge, 2013; Khan et al., 2016; Wiedemann; Meyen, 2016).

Específicamente, Lauf (2005), al explorar la diversidad geográfica de las principales revistas de comunicación, descubrió que los países de habla inglesa, y los Estados Unidos en particular, lideran la producción de las principales revistas. Del mismo modo, Demeter (2019), demostró que las geografías de habla inglesa gobiernan el campo de la comunicación, mientras que las geografías del Sur-Global están en una posición más débil incluso en las ciencias blandas. Finalmente, Demeter (2018), al revisar el estudio de Lauf (2005), sobre la diversidad geográfica de las revistas de comunicación líderes, descubrió que la mayoría de revistas todavía publican investigaciones casi exclusivamente del Norte-Global.

Wiedemann y Meyen (2016, p. 1.489), hallaron patrones de dominación académica similares en el liderazgo de la International Communication Association (ICA), sugiriendo que a pesar de la creciente internacionalización de su liderazgo "el polo de poder del campo sigue siendo una empresa estadounidense". Finalmente, Knobloch-Westerwick y sus colegas hallaron experimentalmente un efecto Matilde tanto en términos de citas como en la percepción de calidad de la investigación. Específicamente, observaron que los artículos de investigación escritos por mujeres tienden a recibir menos citas que los artículos escritos por hombres (Knobloch-Westerwick; Glynn; Huge, 2013), mientras que las publicaciones de autores masculinos tienden a asociarse a una mayor calidad científica, en particular si el tema es de tipo-masculino (Knobloch-Westerwick; Glynn; Huge, 2013). Si bien los diferentes estudios han arrojado luz sobre la composición de los 
consejos editoriales y el desequilibrio geográfico y de género de las principales revistas, el campo de la comunicación todavía está entendiendo cómo estos desequilibrios están estructurados y representados de acuerdo con académicos individuales, sus afiliaciones y geografías (Goyanes, 2017). En la siguiente sección se detallan estas desigualdades en base a la teoría de los Editorial board interlocking y las escuelas invisibles.

\section{Editorial board interlocking y escuelas invisibles}

Como se describió anteriormente, los miembros de los consejos editoriales son académicos que cuentan con una gran reputación y respeto (Teixeira; Oliveira, 2018; Andrikopoulos; Economou, 2015). Como "guardianes del conocimiento" (Metz; Harzing; Zyphur, 2016), determinan lo que se publica y por lo tanto lo que informa el desarrollo de la teoría, la investigación y la práctica (Mauleón et al., 2013; Burgess; Shaw, 2010). Estudios recientes sobre cienciometría (Mauleón et al., 2013; Teixeira; Oliveira, 2018) asumen que los candidatos potenciales a formar parte de un consejo editorial de una revista de primer nivel deben cumplir ciertos criterios, como un sólido historial de publicaciones o una gran experiencia y prestigio. Sin embargo, dada la limitación cuantitativa de tales perfiles y el creciente mecenazgo académico (Bedeian et al., 2009; Burgess; Shaw, 2010), es habitual que un experto asuma una membresía en varios consejos editoriales al mismo tiempo, un fenómeno conocido como editorial board interlocking (Andrikopoulos; Economou, 2015).

Estudios previos han examinado este fenómeno en diferentes disciplinas. En este sentido, existe un consenso tácito sobre sus potenciales efectos en el progreso científico: la membresía cruzada crea una estructura elitista que influye de modo significativo en lo que se publica y, por lo tanto, privilegia el empleo de determinadas metodologías y establece las normas y valores que regulan y gobiernan el sistema de producción (Teixeira; Oliveira, 2018).

Las posibles consecuencias de las membresías cruzadas en los comités científicos también se relacionan con la
Es habitual que un experto asuma una membresía en varios consejos editoria- les al mismo tiempo, un fenómeno co- nocido como editorial board interlocking creación de un subgrupo de académicos vinculados a algunas revistas centrales, ejerciendo así una influencia directa en su visión y paradigmas. Dado que las opiniones, sugerencias, visiones y expectativas de los consejeros editoriales se reflejan en varias revistas a la vez, la teoría sobre editorial board interlocking sugiere que los miembros de estos comités establecen unos patrones de producción/difusión de conocimiento paradigmáticos. Como resultado, las membresías científicas cruzadas tienden a unificar la estrategia y el resultado de la investigación de diferentes revistas en un campo (Teixeira; Oliveira, 2018), creando "colegios invisibles" que establecen sus propias normas y valores (Zuccala, 2006). Estudios anteriores han examinado la membresía cruzada del campo de la estadística (Baccini; Barabesi, 2011), estudios financieros (Andrikopoulos; Economou, 2015), gestión del conocimiento (Teixeira; Oliveira, 2018) o economía (Baccini; Barabesi, 2009). Sin embargo, ningún trabajo previo había examinado este fenómeno en ciencias de la comunicación.

Recientemente, Goyanes y De-Marcos (2020), identifican esta estructura, ofreciendo hallazgos relevantes para explorar su impacto. En primer lugar muestran como los miembros de las revistas en comunicación comparten membresía en varias de ellas, creando unas normas y expectativas compartidas (figura 1). En este caso, se originan varios subgrupos en función del número de miembros que se comparten. El primer subgrupo está conformado por revistas sobre relaciones

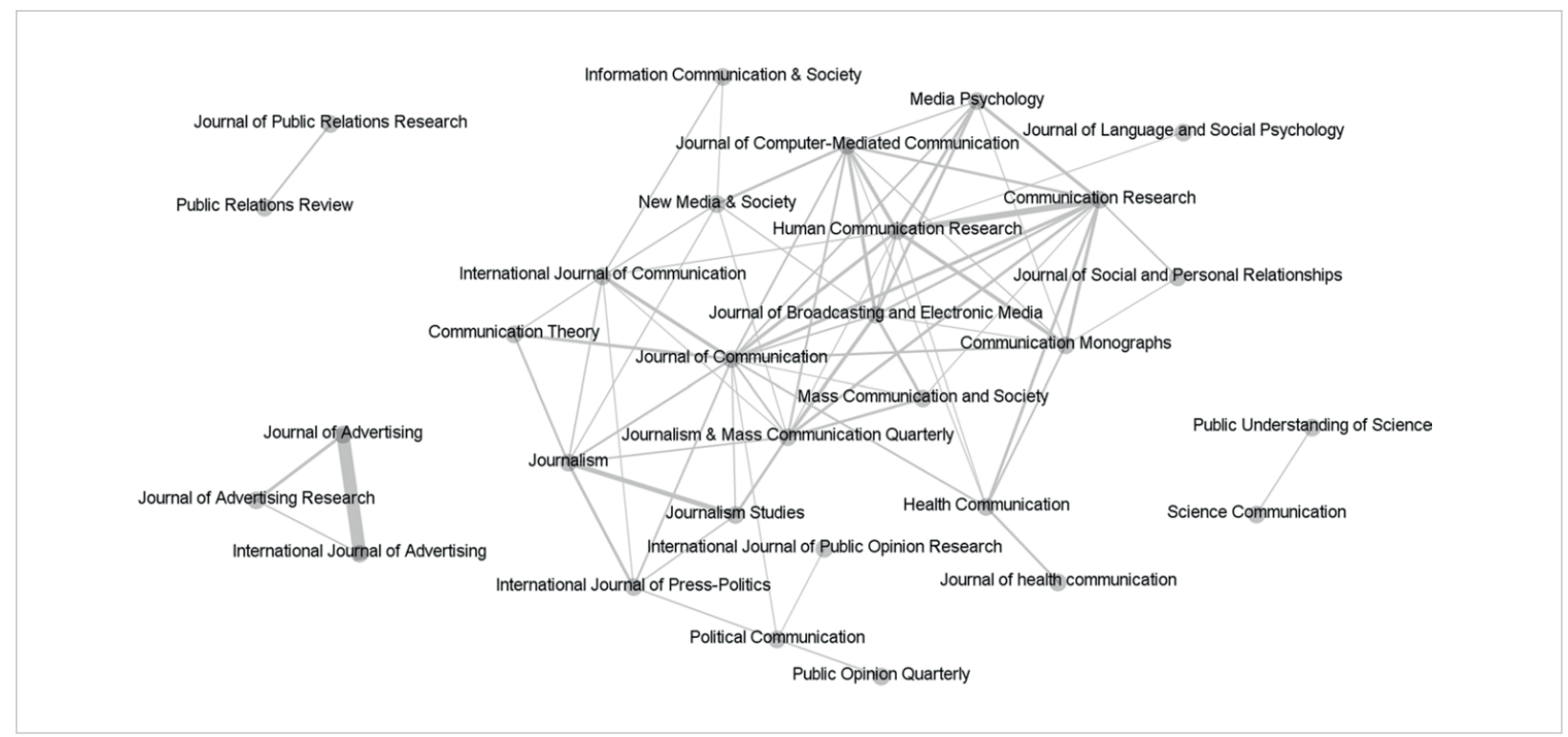

Figura 1. Grupos cohesivos de revistas que comparten cinco o más miembros de sus comités científicos. El grosor de los bordes es proporcional al peso de la conexión. Fuente: Goyanes y De-Marcos (2020). 
públicas e incluye las revistas Journal of public relations research y Public relations review. El segundo subgrupo está formado por revistas de publicidad, englobando a Journal of advertising, Journal of advertising research e International journal of advertising. El tercer subgrupo está formado por dos revistas sobre comunicación de la ciencia, Science communication y Public understanding of science. Finalmente, el último subgrupo es el principal, y está formado por 23 revistas que cubren varias áreas temáticas del campo, incluyendo el periodismo, la comunicación y la comunicación política. Estos cuatro subgrupos comparten al menos cinco editores en sus comités científicos, lo que implica una gran estandarización en las demandas, prioridades y expectativas de producción y evaluación.

La investigación también identifica los grupos cohesivos de académicos basándose en el número de revistas comunes en las que sirven como editores. Por tanto, muestra los perfiles más demandados y la relación que se establece entre ellos. Estos grupos cohesivos de miembros científicos tienen que compartir al menos 4 membresías. Los resultados del análisis de redes muestran la existencia de, al menos, cuatro grupos (figura 2). El primer grupo (a la izquierda en la figura) incluye a 9 académicos, que se centran principalmente en la comunicación en salud. El segundo grupo cohesivo incluye 20 académicos que están conectados por varios temas comunes, incluyendo la communication research, la comunicación política y el periodismo. El tercer grupo cohesivo (parte derecha de la figura) incluye 2 académicos que se centran en los estudios de periodismo. El tamaño y la densidad del subgrupo cohesivo más grande sugiere que estos académicos forman un "colegio invisible", es decir, una élite conectada que desarrolla sus propias normas y valores, monitoreando el progreso del campo (Zuccala, 2006).

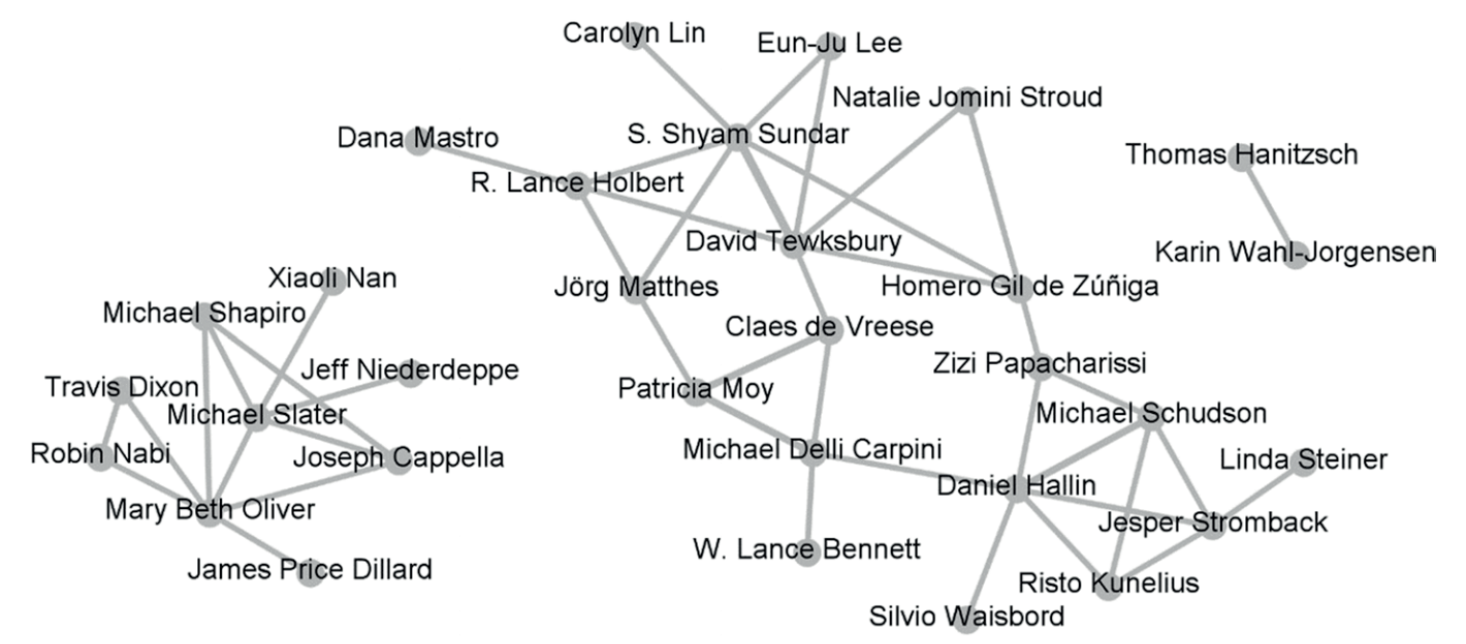

Figura 2. Grupos cohesivos de académicos que forman parte de cuatro o más comités científicos. Fuente: Goyanes y De-Marcos (2020).

\section{Hallazgos y bibliografías clave para dar cuenta de las desigualdades en los estudios en comunicación y sus órganos de gobernanza}

A lo largo de los últimos años, un número creciente de investigadores se ha centrado en examinar las prácticas de investigación en comunicación. El objetivo de esta línea de investigación es la de obtener "conocimiento sobre el conocimiento" y así problematizar los procesos de producción científica y detectar las lagunas de investigación más relevantes (Günther; Domahidi, 2017). En los estudios sobre comunicación internacionales, este "meta-conocimiento" es comúnmente explorado a través de dos perspectivas complementarias que ilustran la identidad, historia, paradigmas y estructura del propio campo: el análisis bibliométrico (Lauf, 2005; Chung et al., 2013; Günther; Domahidi, 2017) y los estudios críticos sobre comunicación (Waisbord; Mellado, 2014; Willemns, 2014).

La investigación sobre bibliometría en ciencias de la comunicación tiene como objetivo obtener conocimiento empírico sobre la evolución del campo mediante el auto-control de los patrones de publicación del material publicado. A pesar de que la mayoría de investigaciones sobre esta línea de investigación se centra en el lado de la oferta de producción (diversidad nacional de autores y coautorías, tendencias de publicación, análisis de citas y redes), sus hallazgos proporcionan evidencias determinantes para ilustrar el estado del campo y, por lo tanto, para calibrar indirectamente la representación distorsionada de los órganos de gobernanza. Diferentes investigaciones han examinado esos patrones en detalle. Por ejemplo, Hugh-Feeley (2008) muestra que Communication monographs, Communication research, Human communication research y Journal of communication son las cuatro revistas más importantes en relación a los patrones de citas entre revistas en comunicación. Igualmente, Barnett y sus colegas (2011), al explorar la relación entre las revistas de comunicación, sugieren que las revistas indexadas únicamente en este campo son más centrales en la red de citas.

Una miríada de estudios ha explorado la conexión estructural entre las teorías del campo en comunicación mediante el análisis de redes. Por ejemplo, Lee, Jung y Song (2016), al explorar la conexión entre temas y métodos, descubrieron que 
la alfabetización mediática es el tema más frecuente y el análisis factorial el método más empleado. Del mismo modo, Chung et al., (2013) sugieren que la teoría del encuadre es la más popular, mientras que Khan et al., (2016) revelan que la investigación y las metodologías innovadoras impulsadas por las teorías de la comunicación no se limitan a esta comunidad científica. Finalmente, Günther y Domahidi (2017) al rastrear el desarrollo de las temáticas de investigación desde los años 30, descubrieron que Internet y las redes sociales se han convertido en las plataformas más populares.

Al igual que en el caso de los análisis bibliométricos, las investigaciones críticas en comunicación han sido muy limitadas. Sobre todo, en relación a la intersección entre poder y conocimiento y, por tanto, sobre las lógicas de dominación y exclusión en los comités científicos. A pesar de esta limitación cuantitativa, los estudios previos se basan en diferentes marcos teóricos para explorar la dinámica Norte-Sur con respecto a las prácticas metodológicas y las normas de producción de conocimiento. En particular, durante la última década, este conjunto de estudios se ha estructurado en torno a discusiones sobre la identidad, los objetivos y los paradigmas de la investigación en comunicación (Craig, 1999; Donsbach, 2006), y la lucha del campo para adoptar una visión de investigación global e internacional (Waisbord; Mellado, 2014). En términos generales, este corpus teórico generalmente se basa en dos marcos críticos e interrelacionados: la bibliografía que razona sobre la globalización de la investigación en comunicación y los estudios poscolonialistas.

Un número progresivo de investigaciones destacó la necesidad de internacionalizar los estudios en comunicación para que sean más representativos de países no-occidentales e impulsar las voces del Sur-Global

Cuando se trata de ilustrar la relación de resistencia/dominación y, por lo tanto, la reproducción de la dinámica núcleo-periferia de la producción de conocimiento, ambas perspectivas proporcionan evidencias determinantes. En este sentido, el número especial de Communication theory (2014, volumen 24) ofrece un enfoque inspirador sobre los crecientes debates alrededor de la "des-occidentalización". Desde su publicación, un número progresivo de investigaciones destacó la necesidad de internacionalizar los estudios en comunicación con el objetivo de 1) construir un campo más representativo en base a las contribuciones de países no-occidentales e 2) impulsar las voces del Sur-Global, reorientando y vigorizando su trabajo intelectual contra el eurocentrismo académico (Waisbord; Mellado, 2014; Willemns, 2014). La des-occidentalización es una llamada a ampliar el análisis, los objetos de estudio, las metodologías y las agendas de investigación desarrolladas en el Norte-Global. La suposición es que las teorías y perspectivas occidentales:

"son inadecuadas para comprender los procesos y fenómenos de comunicación local y regional. Debido a que transfieren categorías y perspectivas extranjeras, la investigación basada en las premisas occidentales conlleva distorsiones ontológicas y analíticas. Por lo tanto, se necesita fortalecer las tradiciones indígenas para superar las limitaciones de los paradigmas occidentales y producir conocimiento que refleje las realidades locales" (Waisbord; Mellado, 2014, pp. 362-363).

Estudios previos han problematizado la relación entre geografía y producción documentando exhaustivamente los potenciales desequilibrios (Murphy; Zhu, 2012; Bell et al., 2017, Goyanes, 2018). La dominación intelectual (a través de la productividad) de los países occidentales no refleja ni la geografía ni la población, sino más bien una distribución global del poder (Bell et al., 2017). En este sentido, la estrecha concordancia entre la distribución del poder académico y económico está bastante bien documentada (Alatas, 2003). Esto no sugiere que haya una ausencia total de estudios no-occidentales en las revistas mejor posicionadas. El conocimiento existente apunta hacia otra dirección: la existencia de un nivel creciente de estudios en las economías emergentes, pero dirigido por autores occidentales (Murphy; Zhu, 2012). Como en las economías capitalistas, los beneficios de la globalización de la investigación se devuelven al Norte-Global.

La globalización de las ciencias sociales también plantea inquietudes sobre la reproducción de las dinámicas núcleo-periferia entre el Norte y el Sur global con respecto a la práctica metodológica y las normas de producción de conocimiento (Gobo, 2011). Investigaciones anteriores han demostrado que la investigación y la publicación en comunicación están dominadas por agendas y orientaciones occidentales que determinan los marcos teóricos y valores epistemológicos, metodológicos y estilísticos (Gunaratne, 2010; Waisbord, 2016). Bajo esta dinámica de dominación académica, algunos observadores han destacado la creciente presión de los investigadores periféricos en "demostrar" su valía científica al ajustarse a las normas y valores de las revistas dominadas por Occidente (Alvesson et al., 2017). La internacionalización, la estandarización y el empirismo se han convertido en las nuevas normas básicas de producción científica (Murphy; Zhu, 2012). El resultado de este proceso es una mayor concentración en los problemas y cuestiones occidentales, la ignorancia de los contextos nacionales y la implementación de marcos y conceptos occidentales para examinar fenómenos locales (Alatas, 2003; Gunaratne, 2010).

La bibliografía poscolonial ofrece una miríada de ejemplos sobre el efecto colonizador de las orientaciones teóricas/metodológicas occidentales en las prácticas de investigación nativas. Por ejemplo, según Ito (1990), los académicos japoneses han apadrinado diferentes puntos de vista sobre la aplicación de la teoría social
Existe un nivel creciente de estudios en las economías emergentes, pero dirigido por autores occidentales 
occidental para examinar los problemas japoneses, en muchos casos traduciendo y aplicando directamente la teoría occidental al japonés. En consecuencia, dentro de las revistas occidentales, los estudios sobre comunicación japoneses se definen y analizan usualmente mediante conceptos occidentales. Por otro lado, las normas de las revistas científicas en comunicación españolas difieren de las de Estados Unidos al hacer mayor énfasis en la interpretación crítica y menos en el empirismo, lo que hace que la producción académica española sea en gran medida "inadmisible" en las llamadas revistas de referencia. Para adaptarse a los cambios recientes en la política científica que obligan a los académicos a publicar en revistas de los Journal Citation Reports (JCR), la comunidad científica se está volviendo cada vez más empirista (Goyanes; Rodríguez-Gómez; Rosique-Cedillo, 2018), adoptando estilos propios de la cultura norteamericana.

\section{Retos y desafíos de la meta-investigación en comunicación en España e internacional}

La problematización de los órganos de gobernanza en comunicación y la revisión de los estudios bibliométricos sobre producción científica ha manifestado intensas desigualdades. A continuación se ofrecen nueve retos clave cuya profundización favorecería el progreso de este programa de investigación. Estos retos o desafíos se sitúan en dos niveles en función del área geográfica: nacional o internacional.

\subsection{Retos nacionales}

\section{Potenciar la estadística inferencial}

La meta-investigación en España está fuertemente vinculada al empleo de la metodología cuantitativa (Carrasco-Campos; Saperas; Martínez-Nicolás, 2018). Sin embargo, la mayoría de estos estudios emplean de modo casi exclusivo la estadística descriptiva (Goyanes; Rodríguez-Gómez; Rosique-Cedillo, 2018), recurriendo a porcentajes y frecuencias a través del empleo generalizado del análisis de contenido. De modo sistemático, estas investigaciones obvian las potenciales relaciones entre variables a través de la estadística inferencial y el empleo de técnicas multivariantes. En general, ello es consecuencia de varios factores estructurantes del sistema académico en comunicación: 1) la preponderancia del pensamiento crítico frente al empirismo 2) el privilegio de la exploración y descripción frente al análisis hipotético-deductivo, 3) el recelo y desconocimiento de la estadística inferencial y 4) la limitación financiera para el diseño de estudios robustos, lo que generalmente desencadena el uso recurrente del análisis de contenido. Estos factores, unidos a la suposición generalizada de que la meta-investigación en comunicación es exclusivamente posible a través del examen de los patrones de producción, provocan que este programa de investigación esté cercano al colapso. En este contexto, muchos investigadores de esta comunidad podrían percibir que la mayoría de variables relevantes ya han sido examinadas con reiterado detalle. Ello genera la impresión de que una buena parte de investigaciones parezcan triviales y redundantes.

Estudios previos ya han manifestado la necesidad de incorporar técnicas y análisis robustos que esbocen hipotéticamente los antecedentes y efectos de los patrones de producción dominantes (origen de los autores, afiliación de procedencia, metodologías empleadas, etc.) y con ello aligerar la redundancia actual. Por tanto, un reto futuro con el que contribuir al avance de este programa de investigación es mediante el planteamiento hipotético-deductivo de relaciones entre las diferentes tendencias de producción que actualmente se encuentran descritas mediante frecuencias y porcentajes.

\section{Estimular la problematización de la cultura y práctica de la publicación}

La meta-investigación sobre comunicación en España toma fundamentalmente como objeto de estudio los patrones de creación científica a través del escrutinio del contenido del material publicado. Ello se produce a consecuencia de la suposición generalizada de que para conocer las tendencias de producción es necesario exclusivamente examinar los artículos científicos. Sin embargo, la creación académica no se sitúa en un vacío, sino que se encaja dentro de un contexto académico, político e institucional que guillotina o asume lo descubierto. La sorprendente carencia de este tipo de investigación oscurece notablemente el progreso de la meta-investigación en España.

Por lo tanto, más allá del examen del contenido publicado, investigaciones futuras deberán profundizar en la cultura de la publicación y las prácticas de investigación que de ella se derivan. Esto incluye, por ejemplo, la presión por publicar, los regímenes de excelencia y sus efectos psicológicos y académicos, la sociología de los

Investigaciones futuras deberán profundizar en la cultura de la publicación y las prácticas de investigación que de ella se derivan departamentos y sus efectos productivos, los efectos institucionales de la evaluación y acreditación científica (Aneca), los efectos de la política científica, la falta de consolidación profesional, los regímenes de indexación, o las prácticas cuestionables de investigación (HARKing, cherry picking, p hacking, etc.).

\section{Examinar las perspectivas y vivencias de los académicos inductivamente}

La meta-investigación en comunicación en España tiende a asumir que para contribuir al conocimiento debe partir exclusivamente del análisis bibliométrico o cienciométrico. Dicho de otro modo, las vivencias y matices de los agentes 
productores (autores) y de la gobernanza (comités científicos), parecen ser poco relevantes para examinar el sistema de producción. Los autores, revisores, teóricos y metodólogos son, sin embargo, los protagonistas del sistema y, por lo tanto, sus perspectivas y vivencias son clave para dar cuenta de la realidad científica. Un reto potencial con el que contribuir al avance de la meta-investigación en España es, en consecuencia, el examen de las perspectivas y vivencias de los agentes productores a través de técnicas de investigación cualitativas, como pueden ser la entrevista en profundidad o el grupo focal, de cada uno de los temas descritos en el punto anterior.

\section{Estudiar los colectivos más desfavorecidos}

Estudios previos sobre las desigualdades en la academia han demostrado que no todos los académicos cuentan con los mismos privilegios a la hora de participar en la ciencia y en sus sistemas de indexación (Bell et al., 2017). Estos factores estructurales y/o coyunturales impactan de modo significativo en la producción científica y en el progreso académico (Alatas, 2003). De modo notable, las universidades con menores recursos, los doctores sin afiliación, los falsos profesores asociados, los académicos sin recursos para el diseño, traducción o edición de artículos científicos, los grupos de investigación sin financiación o simplemente los académicos socializados en lengua española o francesa, cuentan con importantes desafíos más allá de los propios relacionados con el diseño de estudios rigurosos. Muchos de ellos podrían sentirse desplazados en su propio país y con enormes obstáculos para progresar en el cambiante entorno académico.

Estos colectivos, en combinación con las ya documentadas dificultades de las mujeres científicas (Knobloch-Westerwick; Glynn; Huge, 2013), cuentan con significativas desigualdades que deben ser ampliamente documentadas y fuertemente denunciadas. Esos hallazgos pondrían de manifiesto los desequilibrios presentes, ofreciendo resultados basados en evidencias que contribuirían a que las instituciones políticas y sociales establecieran nuevos marcos regulatorios que favorecieran su desempeño profesional.

\subsection{Retos internacionales}

\section{Fomentar las evidencias procedentes del Sur-Global}

Estudios previos en comunicación han demostrado que los autores y fuentes de evidencia de la mayoría de investigaciones proceden de un número muy limitado de países (Livingstone, 2007). El Norte-Global, sobre todo Estados Unidos, Reino Unido, Alemania, Canadá y Australia, encabezan los índices de producción y controlan la mayoría de los órganos de gobernanza (Demeter, 2019). En cierta medida, es natural que estas potencias dominen y establezcan las normas y valores que gobiernan el campo, al ser las geografías que más y mejor invierten en ciencia (Goyanes; De-Marcos, 2020). Sin embargo, la homogeneización geográfica desencadena un proceso de estandarización científica que tiende a oscurecer el pluralismo y la disidencia académica (Goyanes, 2017).
La homogeneización geográfica desen- cadena un proceso de estandarización científica que tiende a oscurecer el plu- ralismo y la disidencia académica

La investigación existente ha documentado detalladamente los patrones de producción del Norte-Global y los efectos de la cultura y política científica. Mediante técnicas multivariantes y métodos inductivos, estos estudios han problematizado la evolución del campo y sus efectos, la interdisciplinariedad, los paradigmas y sus limitaciones así como las prácticas cuestionables de investigación (Donsbach, 2006; Matthes et al., 2015; Walter; Cody; Ball-Rokeach, 2018). Uno de los retos futuros de la meta-investigación en comunicación internacional es la de extender y documentar empíricamente estas temáticas con evidencias del Sur-Global, empleando con mayor asiduidad diferentes técnicas de investigación cualitativas.

\section{Fomentar la apertura intelectual de las revistas y de su sistema de gobernanza}

La publicación internacional de investigaciones rigurosas y ajustadas a las demandas de producción supone un desafío independientemente de la temática. Sin embargo, estudios previos han demostrado las fuertes desigualdades que genera el sistema, lo que provoca el privilegio de determinados fenómenos y aproximaciones metodológicas (Demeter, 2019). En este sentido, la meta-investigación en comunicación es uno de los programas de investigación con mayores prejuicios debido, precisamente, a su propia naturaleza. Tres son los principales obstáculos:

- En primer lugar, la dificultad de establecer el "test de contribución" y de formar una "identidad científica". La meta-investigación, al examinar temáticas que razonan sobre la propia naturaleza del campo, proporciona hallazgos limitados a esta comunidad científica. Asimismo, tienden a adolecer de implicaciones sociales o políticas generales.

- En segundo lugar, y derivada de la anterior, son muy definidos los espacios interesados en estos estudios y su difusión tiende a reducirse a números especiales o revistas especializadas.

- En tercer y último lugar, la sociología y la meta-investigación en las ciencias de la comunicación tratan, entre otras cuestiones, de revelar empíricamente la cultura y práctica científica.

Para los dominadores del campo resulta desafiante que los patrones de producción se manifiesten empíricamente, puesto que se revelan las desigualdades y sus privilegios estructurales 
Para los dominadores del campo resulta desafiante que los patrones de producción se manifiesten empíricamente, puesto que se revelan las desigualdades y sus privilegios estructurales. Ello provoca que muchos académicos reaccionen con recelo y que una parte significativa de estudios críticos sean rechazados en el proceso de revisión por pares. Como antídoto, muchas meta-investigaciones hacen un redoblando esfuerzo de contención crítica, guillotinando su potencial relevancia e interés.

\section{Razonar sobre el modo de reclutamiento de los órganos de gobernanza}

Las investigaciones sobre los consejos científicos asumen que la selección de sus miembros atiende a ideales meritocráticos tales como el historial de publicaciones, la financiación obtenida, los premios concedidos y demás variables perfectamente cuantificables (Dhanani; Jones, 2017). Sin poner en duda las buenas prácticas de la mayoría de revistas, estudios recientes comienzan a desafiar este argumento racional y a teorizar sobre el potencial patronazgo en la ciencia (Burgess; Shaw, 2010).

Dos son los intereses fundamentales:

1) mantener una estructura elitista (colegio invisible) que perpetúe las normas y valores de los dominadores del campo (nivel internacional) y

2) generar influencias o negocios con intereses crematísticos cuya expansión geográfica se basa en la creación y divulgación científica (nivel nacional).

Un reto clave de la meta-investigación es la de examinar estas distorsiones y documentar sus potenciales consecuencias en la producción y promoción académica. Asimismo, estudios futuros deberían profundizar en el rol de facto de determinados perfiles y su contribución al buen gobierno de las revistas a las que pertenecen. Es este sentido, estudios recientes han sugerido que un número significativo de académicos son requeridos en los consejos científicos como mero reclamo, pero con escasa participación estratégica.

\section{Incluir bases de datos más inclusivas (Scopus), frente a Web of Science}

Una parte significativa de la meta-investigación en comunicación toma como referencia para sus análisis las revistas incluidas en la Web of Science de Clarivate Analytics. Ello se debe, principalmente, a que su sistema de clasificación, los Journal Citation Reports, y su "índice de impacto", es considerado el más relevante para clasificar las revistas y su importancia (Goyanes, 2017). A pesar de que existe una fuerte contestación alrededor de este argumento (Demeter, 2018), son muchas las agencias de acreditación y evaluación de todo el mundo que toman esta clasificación como unidad de medida.

Sin embargo, este sistema de indexación ofrece desajustes e imparcialidades que privilegian las costumbres, normas y valores de la cultura anglosajona. Es por ello que los hallazgos obtenidos, si bien pretenden ser generalizables a la población de las revistas en comunicación, contienen grandes limitaciones. Una potencial solución y reto de futuro es el de ampliar la muestra seleccionada y tomar como medida índices de impacto más inclusivos, como SCImago Journal Rank o CiteScore, basados en Scopus. Para reducir la cantidad de material explorado, los estudios deberán basarse en muestras probabilísticas aleatorias, estratificadas por cuartiles y proporcionales. Mediante estás técnicas de muestreo, los hallazgos obtenidos y las implicaciones teóricas discutidas serán perfectamente generalizables y se comprenderá mejor la verdadera evolución del campo.

\section{Plantear nuevas formas de medir la diversidad científica}

Uno de los desafíos clave de la meta-investigación en comunicación es establecer medidas y baremos con los que calcular la diversidad científica. La mayoría de estudios dentro de este programa de investigación ofrecen descripciones de la prevalencia o carencia de determinados valores, como puede ser el número de mujeres que son primeras autoras, las metodologías empleadas, o las universidades y países más productores. En función de sus frecuencias y porcentajes, los investigadores establecen conclusiones teóricas sobre la evolución y devenir del campo, problematizando la potencial diversidad y sus consecuencias. Siendo ellas relevantes, cuentan con importantes limitaciones: ¿bajo qué criterios empíricos se establecen que esas medidas son o no son diversas? ¿cuál es el ratio de diversidad que debe considerarse aceptable? Y lo que es quizá todavía más importante, ¿qué es la diversidad científica y cómo puede medirse matemáticamente? Un reto fundamental de la meta-investigación internacional es proporcionar modelos de medición de la diversidad científica y protocolos fiables para su contraste frente a patrones que se establecen o consideran teóricamente como ideales. Ello permitiría establecer discusiones teóricas con criterios transparentes y el planteamiento de discusiones normativas sobre la verdadera diversidad del campo.
Un reto fundamental de la meta-investigación internacional es proporcionar modelos de medición de la diversidad científica y protocolos fiables 


\section{Referencias}

Alatas, Syed-Farid (2003). "Academic dependency and the global division of labour in the social sciences". Current sociology, v. 51, n. 6, pp. 599-613. https://doi.org/10.1177/00113921030516003

Alvesson, Mats; Gabriel, Yiannis; Paulsen, Roland (2017). Return to meaning: A social science with something to say. Oxford University Press. ISBN: 9780198787099

Andrikopoulos, Andreas; Economou, Labriana (2015). "Editorial board interlocks in financial economics". International review of financial analysis, v. 37, pp. 51-62.

https://doi.org/10.1016/j.irfa.2014.11.015

Baccini, Alberto; Barabesi, Lucio (2010). "Interlocking editorship. A network analysis of the links between economic journals". Scientometrics, v. 82, n. 2, pp. 365-389.

https://doi.org/10.1007/s11192-009-0053-7

Baccini, Alberto; Barabesi, Lucio (2011). "Seats at the table: The network of the editorial boards in information and library science". Journal of informetrics, v. 5, n. 3, p. 382-391.

https://doi.org/10.1016/j.joi.2011.01.012

Barnett, George A.; Huh, Catherine; Kim, Youngju; Park, Han-Woo (2011). "Citations among communication journals and other disciplines: a network analysis". Scientometrics, v. 88, n. 2, pp. 449-469.

https://doi.org/10.1007/s11192-011-0381-2

Bedeian, Arthur G.; Van Fleet, David D.; Hyman III, Hugh (2009). "Scientific achievement and editorial board membership". Organizational research methods, v. 12, n. 2, pp. 211-238.

https://doi.org/10.1177/1094428107309312

Bell, Emma; Kothiyal, Nivedita; Willmott, Hugh (2018). "Methodology-as-Technique and the meaning of rigour in globalized management research". British journal of management, v. 28, n. 3, p. 534-550.

https://doi.org/10.1111/1467-8551.12205

Braun, Tibor; Dióspatonyi, Ildikó (2005). "World flash on basic research: The counting of core journal gatekeepers as science indicators really counts. The scientific scope of action and strength of nations". Scientometrics, v. 62, n. 3, pp. 297-319.

https://doi.org/10.1007/s11192-005-0023-7

Bunz, Ulla (2005). "Publish or perish: A limited author analysis of ICA and NCA journals". Journal of communication, v. 55, n. 4, pp 703-720.

https://doi.org/10.1111/j.1460-2466.2005.tb03018.x

Burgess, Thomas F.; Shaw, Nicola (2010). "Editorial board membership of management and business journals: a social network analysis study of the Financial Times 40". British journal of management, v. 21, n 3, pp. 627-648.

https://doi.org/10.1111/j.1467-8551.2010.00701.x

Butler, Nick; Spoelstra, Sverre (2014). "The regime of excellence and the erosion of ethos in critical management studies". British journal of management, v. 25, n. 3, pp. 538-550.

https://doi.org/10.1111/1467-8551.12053

Cabanac, Guillaume (2012). "Shaping the landscape of research in information systems from the perspective of editorial boards: A scientometric study of 77 leading journals". Journal of the American Society for Information Science and Technology, v. 63, n. 5, pp. 977-996.

https://doi.org/10.1002/asi.22609

Caffarel-Serra, Carmen; Ortega-Mohedano, Félix; Gaitán-Moya, Juan-Antonio (2017). “Investigación en Comunicación en la universidad española en el período 2007-2014". El profesional de la información, v. 26, n. 2, pp. 218-227.

https://doi.org/10.3145/epi.2017.mar.08

Carrasco-Campos, Ángel; Saperas, Enric; Martínez-Nicolás, Manuel (2018). “¿Cómo investigamos la comunicación en España? Universidades públicas y privadas en las publicaciones científicas de comunicación españolas (1990-2014)". AdComunica, n. 15.

http://www.adcomunicarevista.com/ojs/index.php/adcomunica/article/view/457

Chung, Joo-Chung; Barnett, George A.; Kim, Kitae Kim; Lackaff, Derek (2013). "An analysis on communication theory and discipline". Scientometrics, v. 95, n. 3, pp. 985-1002.

https://doi.org/10.1007/s11192-012-0869-4

Craig, Robert (1999). "Communication theory as a field". Communication theory, v. 9, n. 2, pp. 119-161.

https://doi.org/10.1111/j.1468-2885.1999.tb00355.x 
Crane, Diana (1967). "The gatekeepers of science: Some factors affecting the selection of articles of scientific journals". The American sociologist, v. 2, n. 4, pp. 195-201.

https://www.jstor.org/stable/27701277

Demeter, Márton (2018). "Changing center and stagnant periphery in communication and media studies: National diversity of major international journals in the field of communication from 2013 to 2017". International journal of communication, v. 12, pp. 2893-2921.

https://ijoc.org/index.php/ijoc/article/view/9122

Demeter, Márton (2019). "The winner takes it all: International inequality in communication and media studies today". Journalism \& mass communication quarterly, v. 96, n. 1, pp. 37-59.

https://doi.org/10.1177/1077699018792270

Dhanani, Alpa; Jones, Michael (2017). "Editorial boards of accounting journals: gender diversity and internationalisation". Accounting, auditing \& accountability journal, v. 30, n. 5, pp. 1008-1040.

https://doi.org/10.1108/AAAJ-08-2014-1785

Dickersin, Kay; Fredman, Lisa; Flegal, Katherine M.; Scott, Jane D.; Crawley, Barbara (1998). "Is there a sex bias in choosing editors? Epidemiology journals as an example". JAMA, v. 280, n. 3, pp. 260-264.

https://doi.org/10.1001/jama.280.3.260

Donsbach, Wolfgang (2006). "The identity of communication research". Journal of communication, v. 56, n. 3, pp. 437448.

https://doi.org/10.1111/j.1460-2466.2006.00294.x

Espin, Johanna; Palmas, Sebastian; Carrasco-Rueda, Farah; Riemer, Kristina; Allen, Pablo E.; Berkebile, Nathan; Hecht, Kirsten A.; Kastner-Wilcox, Kay; Núñez-Regueiro, Mauricio M.; Prince, Candice; Ríos, Constanza; Ross, Erica; Sangha, Bhagatveer; Tyler, Tia; Ungvari-Martin, Judit; Villegas, Mariana; Cataldo, Tara T.; Bruna, Emilio (2017). "A persistent lack of international representation on editorial boards in environmental biology". PLoS biology, v. 15, n. 12, e2002760. https://doi.org/10.1371/journal.pbio.2002760

Feeley, Thoomas (2008). "A bibliometric analysis of communication journals from 2002 to 2005 ". Human communication research, v. 34, n. 3, pp. 505-520.

https://doi.org/10.1111/j.1468-2958.2008.00330.x

Fernández-Quijada, David; Masip, Pere; Bergillos, Ignacio (2013). "El precio de la internacionalidad: la dualidad en los patrones de publicación de los investigadores españoles en comunicación". Revista española de documentación científica, v. 36, n. 2.

https://doi.org/10.3989/redc.2013.2.936

Fuchs, Christian; Qiu, Jack (2018). "Ferments in the field: Introductory reflections on the past, present and future of communication studies". Journal of communication, v. 68, n. 2, pp. 219-232.

https://doi.org/10.1093/joc/jqy008

García-Carpintero, Esther; Granadino, Begoña; Plaza, Luis (2010). "The representation of nationalities on the editorial boards of international journals and the promotion of the scientific output of the same countries. Scientometrics, v. 84 , n. 3, pp. 799-811.

https://doi.org/10.1007/s11192-010-0199-3

Gobo, Giampietro (2011). "Glocalizing methodology? The encounter between local methodologies". International journal of social research methodology, v. 14, n. 6, pp. 417-437.

https://doi.org/10.1080/13645579.2011.611379

Goyanes, Manuel (2017). Desafío a la investigación estándar en comunicación: Crítica y alternativas. Barcelona: Editorial UOC. ISBN: 9788491166771

Goyanes, Manuel (2019). “Editorial boards in communication sciences journals: Plurality or standardization?”. International communication gazette, v. 82, n. 4, pp. 342-364.

https://doi.org/10.1177/1748048518825322

Goyanes, Manuel; De-Marcos, Luís (2020). “Academic influence and invisible colleges through editorial board interlocking in communication sciences: a social network analysis of leading journals". Scientometrics, v. 123, n. 2, pp. $791-811$. https://doi.org/10.1007/s11192-020-03401-z

Goyanes, Manuel; Demeter, Márton (2020). “How the geographic diversity of editorial boards affects what is published in JCR-ranked communication journals". Journalism \& mass communication quarterly, PrePrint, 26 pp.

https://doi.org/10.1177/1077699020904169 
Goyanes, Manuel; Rodríguez-Gómez, Eduardo-Francisco; Rosique-Cedillo, Gloria (2018). “Investigación en comunicación en revistas científicas en España (2005-2015): de disquisiciones teóricas a investigación basada en evidencias". El profesional de la información, v. 27, n. 5, pp. 1281-1291.

https://doi.org/10.3145/epi.2018.nov.11

Grimes, David; Bauch, Chris T.; Ioannidis, John (2018). “Modelling science trustworthiness under publish or perish pressure". Royal Society Open Science, v. 5, n. 1, pp. 1-19.

https://doi.org/10.1098/rsos.171511

Gunaratne, Shelton (2010). "De-Westernizing communication/social science research: Opportunities and limitations". Media, culture \& society, v. 32, n. 3, pp. 473-500.

https://doi.org/10.1177/0163443709361159

Günther, Elisabeth; Domahidi, Emese (2017). "What communication scholars write about: An analysis of 80 years of research in high-impact journals". International journal of communication, v. 11, pp. 3051-3071.

https://ijoc.org/index.php/ijoc/article/view/6989

Ito, Youichi (1990). "Mass communication theories from a Japanese perspective". Media, culture \& society, v. 12, n. 4, pp. 423-446.

https://doi.org/10.1177/016344390012004002

Keating, David M.; Richards, Adam S.; Palomares, Nicholas A.; Banas, John A.; Joyce, Nick; Rains, Stephen A. (2019). "Titling practices and their implications in communication research 1970-2010: Cutesy cues carry citation consequences". Communication research, 22 pp., preprint.

https://doi.org/10.1177/0093650219887025

Khan, Gohar-Feroz; Lee, Sungjoon; Park, Ji-Young; Park, Han-Woo (2016). "Theories in communication science: a structural analysis using webometrics and social network approach". Scientometrics, v. 108, n. 2, pp. 531-557.

https://doi.org/10.1007/s11192-015-1822-0

Knobloch-Westerwick, Silvia; Glynn, Carroll; Huge, Michael (2013). "The Matilda effect in science communication: an experiment on gender bias in publication quality perceptions and collaboration interest". Science communication, v. 35 , n. 5, pp. 603-625.

https://doi.org/10.1177/1075547012472684

Lauf, Edmund (2005). "National diversity of major international journals in the field of communication". Journal of communication, v. 55, n. 1, pp. 139-151.

https://doi.org/10.1111/j.1460-2466.2005.tb02663.x

Livingstone, Sonia (2007). "Internationalizing media and communication studies: reflections on the International Communication Association". Global media and communication, v. 3, n. 3, pp. 273-288.

https://doi.org/10.1177/1742766507082570

Martínez-Nicolás, Manuel; Saperas, Enric (2016). “Objetos de estudio y orientación metodológica de la reciente investigación sobre comunicación en España (2008-2014) Análisis de los trabajos publicados en revistas científicas españolas”. Revista latina de comunicación social, n. 71, pp. 1365-1384.

https://doi.org/10.4185/RLCS-2016-1150es

Martínez-Nicolás, Manuel; Saperas, Enric; Humanes, María-Luisa (2019). “Mudanzas en la cultura científica. El nuevo contexto de la práctica investigadora sobre comunicación y sus implicaciones epistemológicas". En: Epistemología de la comunicación y cultura digital: Retos emergentes (pp. 23-42). Universidad de Granada. ISBN: 9788433865274 https://dialnet.unirioja.es/servlet/libro?codigo=740913

Matthes, Jörg; Marquart, Franziska; Naderer, Brigitte; Arendt, Florian; Schmuck, Desirée; Adam, Karoline (2015). "Questionable research practices in experimental communication research: A systematic analysis from 1980 to 2013 ". Communication methods and measures, v. 9, n. 4, pp. 193-207.

https://doi.org/10.1080/19312458.2015.1096334

Mauleón, Elba; Hillán, Laura; Moreno, Luz; Gómez, Isabel; Bordons, María (2013). “Assessing gender balance among journal authors and editorial board members". Scientometrics, v. 95, n. 1, pp. 87-114.

https://doi.org/10.1007/s11192-012-0824-4

Metz, Isabel; Harzing, Anne-Wil (2009). "Gender diversity in editorial boards of management journals". Academy of Management Learning \& Education, v. 8, n. 4, pp. 540-557.

https://doi.org/10.5465/amle.8.4.zqr540

Metz, Isabel; Harzing, Anne-Wil; Zyphur, Michael (2016). "Of journal editors and editorial boards: who are the trailblazers in increasing editorial board gender equality?" British journal of management, v. 27, n. 4, pp. 712-726.

https://doi.org/10.1111/1467-8551.12133 
Miconi, Andrea; Serra, Marcello (2019). "On the concept of medium: An empirical study". International journal of communication, v. 13, 344-3461.

https://ijoc.org/index.php/ijoc/article/view/10751

Morton, Melinda; Sonnad, Seema (2007). "Women on professional society and journal editorial boards". Journal of the National Medical Association, v. 99, n. 7, pp. 764-771.

https://www.ncbi.nlm.nih.gov/pmc/articles/PMC2574346/

Murphy, Jonathan; Zhu, Jingqi (2012). "Neo-colonialism in the academy? Anglo-American domination in management journals". Organization, v. 19, n. 6, pp. 915-927.

https://doi.org/10.1177/1350508412453097

Ozbilgin, Mustafa (2004). “International” human resource management. Personnel review, v. 33, n. 2, pp. $205-221$. https://doi.org/10.1108/00434804105180559

Parker, Lee (2007). "Developing research journals and qualitative inquiry: the role of the editorial board". Qualitative research in accounting \& management, v. 4, n. 3, pp. 168-182.

https://doi.org/10.1108/11766090710826628

Pendlebury, David; Adams, Jonathan (2012). "Comments on a critique of the Thomson Reuters journal impact factor". Scientometrics, v. 92, n. 2, pp. 395-401.

https://doi.org/10.1007/s11192-012-0689-6

Petersen, Jessica; Hattke, Fabian; Vogel, Rick (2017). “Editorial governance and journal impact: a study of management and business journals". Scientometrics, v. 112, n. 3, pp. 1593-1614.

https://doi.org/10.1007/s11192-017-2434-7

Rodrigo-Alsina, Miguel; García-Jiménez, Leonarda (2010). “Communication theory and research in Spain: A paradigmatic case of a socio-humanistic discipline". European jour- nal of communication, v. 25, n. 3, pp. 273-286.

https://doi.org/10.1177/0267323110373458

Sidhu, Reena; Rajashekhar, Praveen; Lavin, Victoria L.; Parry, Joanne; Attwood, James; Holdcroft, Anita; Sanders, David S. (2009). "The gender imbalance in academic medicine: a study of female authorship in the United Kingdom". Journal of the Royal Society of Medicine, v. 102, n. 8, pp. 337-342.

https://doi.org/10.1258/jrsm.2009.080378

Stegmaier, Mary; Palmer, Barbara; Van Assendelft, Laura (2011). "Getting on the board: the presence of women in political science journal editorial positions". PS: Political science \& politics, v. 44, n. 4, pp. 799-804.

https://doi.org/10.1017/S1049096511001284

Teixeira, Eduardo; Oliveira, Mirian (2018). "Editorial board interlocking in knowledge management and intellectual capital research field". Scientometrics, v. 117, n. 3, pp. 1853-1869.

https://doi.org/10.1007/s11192-018-2937-x

Waisbord, Silvio (2016). "Communication studies without frontiers? Translation and cosmopolitanism across academic cultures". International journal of communication, v. 10, pp. 868-886.

https://ijoc.org/index.php/ijoc/article/view/3483/1569

Waisbord, Silvio; Mellado, Claudia (2014). “De-westernizing communication studies: A reassessment". Communication theory, v. 24, n. 4, pp. 361-372.

https://doi.org/10.1111/comt.12044

Walter, Nathan; Cody, Michael; Ball-Rokeach, Sandra (2018). "The ebb and flow of communication research: Seven decades of publication trends and research priorities". Journal of communication, v. 68, n. 2, pp. 424-440.

https://doi.org/10.1093/joc/jqx015

Wiedemann, Thomas; Meyen, Michael (2016). "Internationalization through Americanization: The expansion of the international communication association's leadership to the world". International journal of communication, v. 10, pp. 1489-1509.

https://ijoc.org/index.php/ijoc/article/view/4504

Willett, Peter (2013). "The characteristics of journal editorial boards in library and information science". International journal of knowledge content development \& technology, v. 3, n. 1, pp. 5-17.

https://journals.sfu.ca/ijkcdt/index.php/ijkcdt/article/view/28

Zuccala, Alesia (2006). "Modeling the invisible college". Journal of the American Society for information Science and Technology, v. 57, n. 2, pp. 152-168.

https://doi.org/10.1002/asi.20256 